\title{
Linear sweep voltammetry with non-triangular waveforms at a microdisc electrode
}

\author{
Yuki Uchida, Enno Kätelhön, and Richard G. Compton \\ Department of Chemistry, Physical and Theoretical Chemistry Laboratory, Oxford University, South Parks \\ Road, Oxford, OX1 3QZ, United Kingdom
}

\begin{abstract}
The voltammetry of an electrochemically reversible reaction at a microdisc electrode is investigated for a [text deleted] semi-circular potential waveform. [text deleted] At the midpoint of the potential window [text deleted] an instantaneous infinite scan rate is observed whilst relatively [text deleted] slow scan rates are seen in other areas of the wave. As a result, the method exhibits a much higher current response than the conventional triangular potential wave thus allowing qualitative analysis of a species with significantly greater ease, particularly at low analyte concentrations in solutions comprising multiple analytes.
\end{abstract}

\section{Introduction}

Linear sweep voltammetry is commonly performed through the application of a linear potential wave ${ }^{1}$, where the scan rate is constant throughout the sweep. The resulting voltammograms give information regarding the redox system under investigation such as analyte concentrations, diffusion constants, and rate constants ${ }^{2 ; 3}$. The linear potential wave is given as:

$$
E(t)= \pm \nu t+E(t=0)
$$

where $E(t)$ is the applied potential with respect to time, \pm sign shows whether the reaction is oxidative or reductive, and $\nu$ is the scan rate. In our previous work ${ }^{4}$ we have introduced a new method for carrying out linear sweep voltammetry at a macroelectrode using a semi-circular potential wave, which creates a transiently infinitely fast scan rate at the midpoint of the potential window and slower scan rates everywhere else. The infinitely fast scan rate results in a significant increase in the current response while the slow scan rates in other areas reduce the current response. Through analysis we have shown that our new method is useful in electroanalytical applications and can be used for the selective detection of the presence of a species in a mixture.

In this paper we further develop the concept and investigate the voltammetric response at a microdisc electrode when employing a semi-circular potential wave. Microelectrodes are widely used due to their advantages over macroelectrodes such as the reduced capacitance and ohmic drop, increased rates of mass transport, fast response time, and a steady-state current reponse ${ }^{1 ; 5-10}$. Our analysis shows that the microdisc electrode shows the emergence of an "infinite" peak current at the potential where the 
singularity in the scan rate is observed and the suppression of the current response in other potential regions. These advantages make the semi-circular potential wave an advantageous tool for electroanalysis.

\section{Theory}

We herein introduce the theoretical model employed and explain the semi-circular potential waveform used in our simulation, and further introduce the dimensionless coordinates as well. Details of the simulations are also presented.

\section{$2.1 \quad$ Theoretical model}

We study a simple one-electron electrochemically-reversible reduction reaction of $A$ to $B$ at a microdisc electrode under diffusion-only conditions, where species $A$ and $B$ have equal diffusion coefficients:

$$
A+e \rightleftharpoons B
$$

The above system is simulated with diffusion as the only means of mass transport of analyte to the electrode surface ${ }^{11-13}$ under the assumption of sufficiently high concentration of supporting electrolyte in order to suppress migration $4 ; 10 ; 14 ; 15$. However, when the scan duration is long, convection ${ }^{16}$ should be taken into consideration ${ }^{7 ; 17-19}$. The transport of analyte is predicted by Fick's second law expressed as $^{1 ; 20 ; 21}$ :

$$
\frac{\partial c_{j}}{\partial t}=D_{j}\left(\frac{\partial^{2} c_{j}}{\partial x^{2}}+\frac{\partial^{2} c_{j}}{\partial y^{2}}+\frac{\partial^{2} c_{j}}{\partial z^{2}}\right)
$$

where $j$ is the species under study and $D_{j}$ is its diffusion coefficient. For a microelectrode simulation where the diffusion is not always planar, Equation (3) must be written in cylindrical polar coordinates giving:

$$
\frac{\partial c_{j}}{\partial t}=D_{j}\left(\frac{\partial^{2} c_{j}}{\partial r^{2}}+\frac{1}{r} \frac{\partial c_{j}}{\partial r}+\frac{1}{r^{2}} \frac{\partial^{2} c_{j}}{\partial \phi^{2}}+\frac{\partial^{2} c_{j}}{\partial z^{2}}\right)
$$

where $z$ is the perpendicular distance from the electrode surface, $r$ is the radial distance from the axis, and $\phi$ is the angle around the axis ${ }^{1 ; 20}$. Since concentration does not change with the angle, $\phi$, Equation (4) is reduced to ${ }^{1 ; 20 ; 22 ; 23}$ :

$$
\frac{\partial c_{j}}{\partial t}=D_{j}\left(\frac{\partial^{2} c_{j}}{\partial r^{2}}+\frac{1}{r} \frac{\partial c_{j}}{\partial r}+\frac{\partial^{2} c_{j}}{\partial z^{2}}\right)
$$

\subsection{Boundary conditions}

For a system using a macroelectrode, only linear diffusion is observed and thus we need only consider the concentration change in the $x$ direction, bounded by the electrode surface and the outer surface. However, microelectrodes introduce non-planar diffusion and we thus need to consider the concentration in the $z$ and $r$ directions, resulting in a two-dimensional rectangular simulation space ${ }^{20}$. Assuming the microelectrode is embedded in a planar supporting surface, the boundary line in the $z$ direction is separated into two parts: one where it is bounded by the electrode surface and the outer surface, and the other bounded by the insulating supporting surface and the outer surface. 
Given $D_{A}=D_{B}$, the following boundary conditions can be established under the assumption that initially only the reactant is present and prior to the experiment the concentration of reactant and product are uniform in space ${ }^{1 ; 20}$ :

$$
t \leq 0 ; \text { all } z, r ; c_{A}=c_{A}^{*} ; c_{B}=0
$$

With increasing time, the concentration near the electrode surface varies but at the outer boundary surface no change in concentration is observed:

$$
\begin{aligned}
& t>0, z \rightarrow \infty, c_{A}=c_{A}^{*}, c_{B}=0 \\
& t>0, r \rightarrow \infty, c_{A}=c_{A}^{*}, c_{B}=0
\end{aligned}
$$

We further introduce two no-flux boundaries, one at the insulating supporting surface, and the other at the cylindrical axis, $r=0$. At a no-flux boundary, no change in concentration gradient is observed giving:

$$
\begin{gathered}
t>0, r>\epsilon,\left(\frac{\partial c}{\partial z}\right)_{z=0}=0 \\
t>0, \text { all } z,\left(\frac{\partial c}{\partial r}\right)_{r=0}=0
\end{gathered}
$$

where $\epsilon$ is the radius of the electrode. When the diffusion coefficients of the reactants and products are equal, conservation of mass predicts that the amount of $A$ consumed at the electrode surface equals the amount of $B$ produced:

$$
t>0, z=0,-\frac{\partial c_{A}}{\partial z}=\frac{\partial c_{B}}{\partial z}
$$

and the following relation holds true for all space and time:

$$
c_{A}+c_{B}=c_{A}^{*}
$$

The electrode kinetics is described through the Butler-Volmer equation given as:

$$
j=k^{0} \exp \left[\frac{-\alpha_{A / B} F\left(E-E_{f, A / B}^{0}\right)}{R T}\right][A]_{0}-k^{0} \exp \left[\frac{\left(1-\alpha_{A / B}\right) F\left(E-E_{f, A / B}^{0}\right)}{R T}\right][B]_{0}
$$

where $j$ is the flux of the reactant to the electrode surface, $k^{0}$ is the standard electrochemical rate constant, $[A]_{0}$ and $[B]_{0}$ are the surface concentrations of species $A$ and $B$ respectively, $\alpha$ is the transfer coefficient ${ }^{24 ; 25}, F, R$, and $T$ hold their conventional significance, $E$ is the applied potential, and $E_{f, A / B}^{0}$ is the formal potential of the $A / B$ redox couple. The flux, $j$, is also described via Fick's first law. Assuming equal diffusion coefficients, $D$, it can be written as:

$$
j=-D\left(\frac{\partial c_{A}(z, t)}{\partial z}\right)_{z=0}=-\left[k_{r e d} c_{A}(0, t)-k_{o x} c_{B}(0, t)\right]
$$

and by subsituting the relationships $\theta=\frac{F}{R T}\left(E-E_{f, A / B}^{0}\right), k_{r e d}=k^{0} e^{-\alpha \theta}$, and $k_{o x}=k^{0} e^{(1-\alpha) \theta}$, Equation (14) becomes: 


$$
D\left(\frac{\partial c_{A}(z, t)}{\partial z}\right)=k^{0} e^{-\alpha \theta} c_{A}(0, t)-k^{0} e^{(1-\alpha) \theta} c_{B}(0, t)
$$

Our system of interest was investigated under the assumption the $A / B$ couple is fully reversible by applying a very large $k^{0}$ value to the Butler-Volmer boundary condition, and hence Equation (13) becomes indistinguishable from the Nernst equation giving ${ }^{20 ; 26}$ :

$$
E=E_{f, A / B}^{0}+\frac{R T}{F} \ln \frac{[A]_{0}}{[B]_{0}}
$$

A related convergence test to ensure the reversibility of the system with the $k^{0}$ value used in this paper is given in the Supporting Information under the heading "Determination of the appropriate $K^{0}$ ", which was conducted using the Butler-Volmer boundary conditions as above.

\subsection{Dimensionless coordinates}

All simulations were run using the dimensionless coordinates of the equations given earlier and their conversion factors are listed in Table 1.

Table 1: Dimensionless parameters. ${ }^{20}$

\begin{tabular}{ll}
\hline Parameter & Normalization \\
\hline concentration & $C_{j}=\frac{c_{j}}{c_{A}^{*}}$ \\
diffusion coefficient & $d_{j}=\frac{D_{j}}{D_{A}}$ \\
z spatial coordinate & $Z=\frac{z}{\epsilon}$ \\
r spatial coordinate & $R=\frac{r}{\epsilon}$ \\
time & $\tau=\frac{D_{A} t}{\epsilon^{2}}$ \\
potential & $\theta=\left(\frac{F}{R T}\right)\left(E-E_{f, A / B}^{0}\right)$ \\
scan rate & $\sigma=\left(\frac{\epsilon^{2}}{D_{A}}\right)\left(\frac{F}{R T}\right) \nu$ \\
current & $J=\frac{I}{2 \pi \epsilon F D_{A} c_{A}^{*}}$ \\
rate constant & $K^{0}=\frac{k^{0} \epsilon}{D_{A}}$ \\
\hline
\end{tabular}

Using these conversion factors, Fick's second law can be expressed in its dimensionless form:

$$
\frac{\partial C}{\partial T}=d_{j}\left(\frac{\partial^{2} C}{\partial R^{2}}+\frac{1}{R} \frac{\partial C}{\partial R}+\frac{\partial^{2} C}{\partial Z^{2}}\right)
$$

and Fick's first law can be rewritten as:

$$
\frac{C_{A, 1}-C_{A, 0}}{h}=\frac{1}{d_{j}} K_{0} f(\theta)\left\{C_{A, 0}-\left[1+C_{B}^{*}-C_{A, 0}\right] e^{\theta}\right\}
$$

where $C_{A, 1}$ and $C_{A, 0}$ are the dimensionless concentrations of species $A$ at position $Z_{1}$ and $Z_{0}$ respectively, $f(\theta)=e^{-\alpha \theta}$, and $h=Z_{1}-Z_{0}$ assuming $A$ and $B$ have equal diffusion coefficients. 


\subsection{Semi-circular potential wave}

In this paper we employ the semi-circular potential waveform and the conventional triangular waveform (shown in Equation(1)). The equation used to describe the semi-circular potential waveform is ${ }^{4}$ :

$$
\theta(\tau)= \pm \sqrt{\left|A^{2}-\left[\sigma_{a v g} \cdot\left(\tau-c \tau_{\max }\right)\right]^{2}\right|}+\theta_{\text {shift }}
$$

where $\theta(\tau)$ is the dimensionless potential with respect to dimensionless time, $A$ is the amplitude, $\sigma_{a v g}$ is the dimensionless average scan rate, $c$ is the fraction of the maximum time when the centre of each semi-circle is observed, $\tau_{\max }=\frac{2 A}{\sigma_{a v g}}$, and $\theta_{\text {shift }}$ is the potential shift defined as $\theta_{\text {shift }}=\theta_{\text {avg }}-\theta_{f, A / B}^{0}$. From Table $1, \theta_{f, A / B}^{0}$ is by definition equal to 0 and hence $\theta_{\text {shift }}=\theta_{\text {avg }}$. For a more detailed discussion on the derivation of Equation (19), please refer to our previous paper ${ }^{4}$. The key feature of the semicircular potential wave is that it produces an instantaneously infinite scan rate at the mid-point of the wave and a slower scan rate at all other areas thereby amplifying the current response between a very narrow potential range and suppressing it at other potential regions, making it suitable for analytical applications.

Figure $1^{4}$ shows the conventional triangular and the [text deleted] semi-circular potential wave centred at $\theta=0$ with $A=1$. For this figure, the first half of the semi-circular potential wave was plotted using $c=0$ and the second half was plotted using $c=1$. Since the scan rate is defined as the slope of the potential wave, as can be seen, the scan rate for the triangular potential wave is constant throughout the entire sweep. On the other hand, the semi-circular potential wave shows significant changes throughout the sweep. At $t=0$ and $t=t_{\max }$, the scan rates are close to zero but at $\theta=0$, the instantaneous scan rate approaches infinity due to the infinite value of $\frac{\partial \theta}{\partial \tau}$. It is known through conventional CV and LSV that increased scan rates lead to greater flux ${ }^{1}$ and thus the transiently infinitely fast scan rate arising from the semi-circular potential wave will result in an infinite current response if the infinitely fast scan rate is reached near the formal potential.

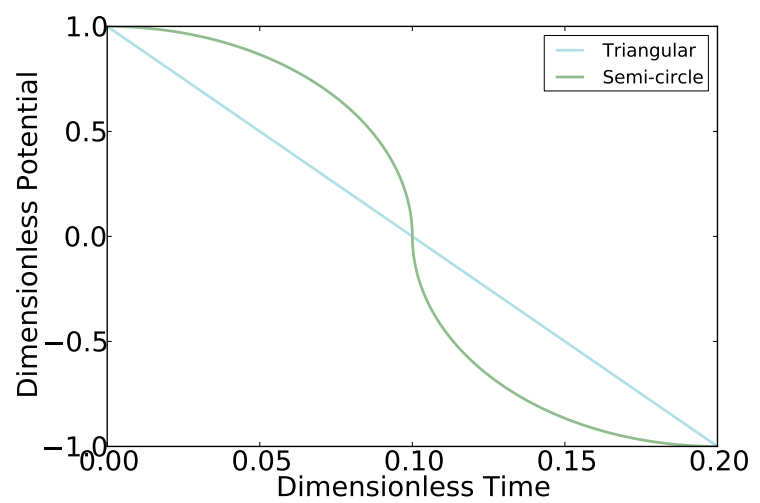

Figure 1: Conventional triangular and semi-circular potential wave graphs centered at $\theta=0$ with an amplitude of 1 . The triangular potential wave shows a constant scan rate throughout the duration time whereas the semi-circular potential wave shows very slow scan rates at $t=0$ and $t=t_{\max }$, but then increases rapidly near $\theta=0$. The figure was directly sited from ${ }^{4}$.

\subsection{Simulation via finite differences}

All results were obtained through the application of the ADI (alternating direction implicit) method by discritizing the dimensionless diffusion equation to solve the system ${ }^{20 ; 27 ; 28}$. The simulation was performed 
in $C++$ with OpenMP for parallel simulation while the plotting was done in Python using packages Numpy and matplotlib. We understand the importance of thorough testing ${ }^{29}$ and have compared the peak heights with theoretical values and have performed convergence tests.

\section{Results and discussion}

The results are divided into two sections in which we first present results from both, the triangular and semi-circular potential waves at three different scan rates of $\sigma=10^{3}, 10^{1}$, and $10^{-1}$, and second we discuss the voltammetric features obtained from the semi-circular potential wave when $\theta_{\text {shift }}$ is varied. For all results shown, the formal potential of the $A / B$ couple is set to 0 , the transfer coefficient, $\alpha$, is set to 0.5 and the dimensionless standard electrochemical rate constant, $K^{0}$ is set to $10^{10}$ to ensure full reversibility of the redox system.

\subsection{Triangular versus semi-circular potential sweeps: 3 different scan rates}

We first focus on the voltammetric differences shown between the triangular and semi-circular potential wave at three different scan rates of $\sigma=10^{3}, 10^{1}$, and $10^{-1}$. Figure 2 shows the voltammetric response from both, triangular and semi-circular potential waves at $\sigma=10^{3}$ and $\theta_{\text {shift }}=0$. The blue solid line represents the result obtained from the triangular potential wave and the green solid line represents the results obtained from the semi-circular potential wave. The red solid line represents the semi-empirical peak height of a reversible system at a microelectrode using a triangular potential wave predicted by the following empirical expression ${ }^{20}$ :

$$
I_{p}=-4 F \epsilon c_{j}^{*} D_{j}\left(0.34 e^{-0.66 \sqrt{\sigma}}+0.66-0.13 e^{-11 / \sqrt{\sigma}}+0.351 \sqrt{\sigma}\right)
$$

Under conditions where $\sigma \rightarrow 0$, Equation (20) simplifies to:

$$
I_{l i m, s s}=-4 F \epsilon c_{j}^{*} D_{j}
$$

where steady-state limiting current is observed and is represented as a pink dotted line in Figure 2. The orange dotted line represents the peak current of a reversible system at a macroelectrode predicted by the Randles-Ševčík equation ${ }^{30 ; 31}$ given as:

$$
I_{p}=-0.446 F A c_{j}^{*} \sqrt{\frac{F D_{j} \nu}{R T}}
$$

where $A$ is the area of the electrode. The arrow shows the direction of the sweep. For all results plotted, the dimensionless forms of Equations (20), (21), and (22) were used, giving the following three equations respectively:

$$
\begin{gathered}
J_{p}=-\frac{2}{\pi} C_{j}^{*} d_{j}\left(0.34 e^{-0.66 \sqrt{\sigma}}+0.66-0.13 e^{-11 / \sqrt{\sigma}}+0.351 \sqrt{\sigma}\right) \\
J_{l i m, s s}=-\frac{2}{\pi} C_{j}^{*} d_{j} \\
J_{p}=-0.223 C_{j}^{*} \sqrt{d_{j} \sigma}
\end{gathered}
$$




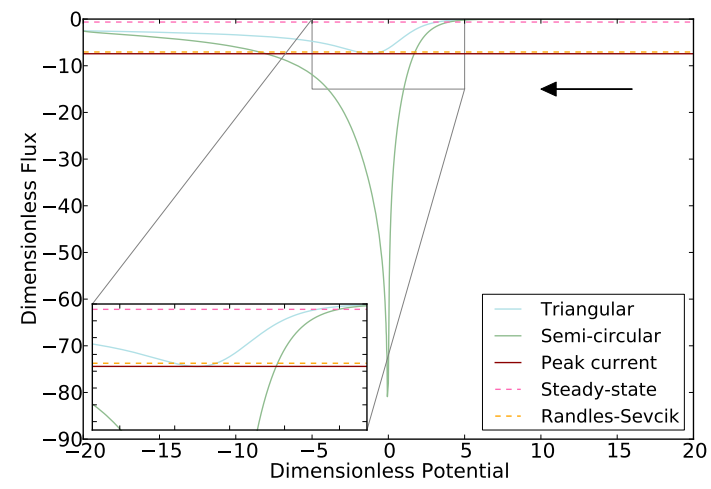

Figure 2: Voltammetric response using triangular and semi-circular potential waves at $\sigma=10^{3}$. The blue solid line represents the result obtained from the triangular potential wave and the green solid line represents the results obtained from the semi-circular potential wave. The red solid line represents the theoretical peak height of a reversible system at a microelectrode using a triangular potential wave predicted by Equation (23), the pink dotted line represents the steady-state limiting current predicted by Equation (24), and the orange dotted line represents the peak current of a reversible system at a macroelectrode predicted by Equation (25). The arrow indicates the direction of the sweep.

From immediate observation, it is clear that the results from the semi-circular potential wave has a significantly larger peak current compared to that from the triangular potential wave, and the peak current approaches infinity at $\theta=0$ and rapidly falls off in all other areas as predicted earlier. In Figure 2 the peak current from the triangular potential wave matches the value predicted by Equation (23) but differs slightly from that predicted by Equation (25). This is because although the scan rate is relatively fast, it is not fast enough to converge the two expressions. Later we investigate cases with higher scan rates and show that at very fast scan rates, Equations (23) and (25) predict the same value.

Figure 3 again shows the voltammetric response from both, triangular and semi-circular potential waves but at a different scan rate of $\sigma=10^{1}$ while maintaining the same potential shift value of $\theta_{\text {shift }}=0$. The same color scheme is used as in Figure 2 . Here we see the current response is reduced by approximately a tenth, but the overall behavior is the same as in Figure 2 with the semi-circular potential wave result showing significantly higher peak current at $\theta=0$ and relatively low currents everywhere else. Figure 3 also shows that the peak height obtained from the triangular potential wave diverges further from the value predicted by the Randles-Ševčík equation due to the slower scan rate, and instead, approaches a steady-state as can be seen from closeness between the red solid line and pink dotted line.

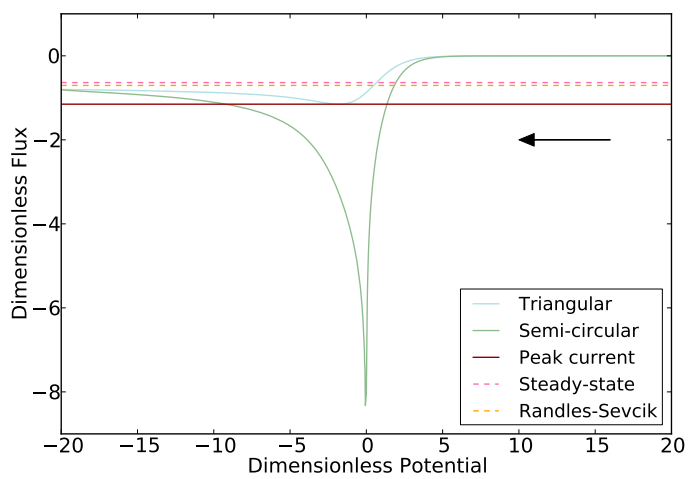

Figure 3: Voltammetric response using triangular and semi-circular potential waves at $\sigma=10^{1}$. See caption of Figure 2 for further details. 
Figure 4 shows the triangular and semi-circular potential wave current response at $\sigma=10^{-1}$ and $\theta_{\text {shift }}=0$. Again, the same color scheme is used as in Figures 2 and 3. The result from the triangular potential wave shows a near steady-state response with little visible peak, and the red solid line is nearly converged with the pink dotted line, confirming the near steady-state limit. The result from the semicircular potential wave is similar to that from the triangular potential wave and can be seen approaching a steady-state limit but still shows a sharp peak at $\theta=0$ although the value is much smaller compared to that seen in Figure 2.

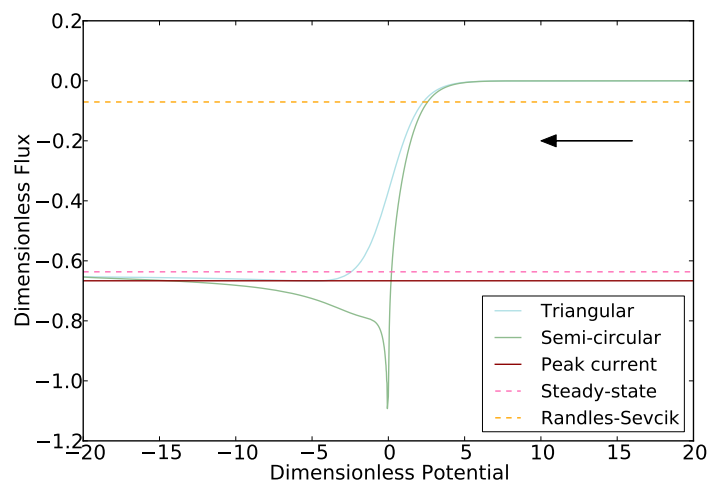

Figure 4: Voltammetric response using triangular and semi-circular potential waves at $\sigma=10^{-1}$. See caption of Figure 2 for further details.

We have focused on results using scan rates of $10^{3}, 10^{1}$, and $10^{-1}$ because interesting voltammetric features arise from the semi-circular potential wave at the transitioning stage from a fully voltammetric response to a fully steady-state limit. Below we show two extreme cases, one when the scan rate is very fast and one when it is very slow.

Figure 5 shows the voltammetric response using the triangular and semi-circular potential wave at $\sigma=10^{8}$ and $\theta_{\text {shift }}=0$ with the same color scheme as Figures 5, 3, and 4. As discussed earlier, when $\sigma \rightarrow \infty$, a planar diffusion is observed ${ }^{1 ; 32}$ at the electrode surface, resulting in a macroelectrode cyclic voltammetry behavior. As a result, Equation (23) converges with the Randles-Ševčík expression and predict the same peak height value, which can be seen through the perfect overlap of the red solid line and orange dotted line in Figure 5. The voltammetric features observed here are mostly identical to that in Figure 2, except the current response is much larger in Figure 5 due to the faster scan rate. 


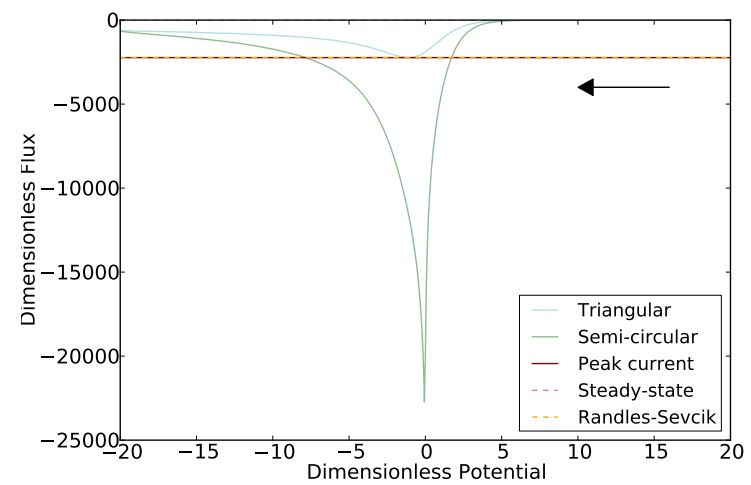

Figure 5: Voltammetric response using triangular and semi-circular potential waves at $\sigma=10^{8}$. See caption of Figure 2 for further details.

Figure 6 shows the voltammetric response of the triangular and semi-circular potential wave at $\sigma=$ $10^{-4}$ and $\theta_{\text {shift }}=0$ again using the same color scheme as Figures 2, 3, 4, and 5 . The current shows a full steady-state behavior and Equations (23) and (24) predict the same value, which can be seen through the overlap of the red solid line and the pink dotted line. Under full steady-state conditions, current is independent of the scan rate ${ }^{33}$ and hence the transiently infinite scan rate produced by the semi-circular potential wave does not show much effect on the current response compared to that obtained from a triangular potential wave, producing nearly identical curves as evident from the overlap of the blue and green solid lines in Figure 6. In such cases, the semi-circular potential wave does not hold any advantage over the conventional triangular potential wave.

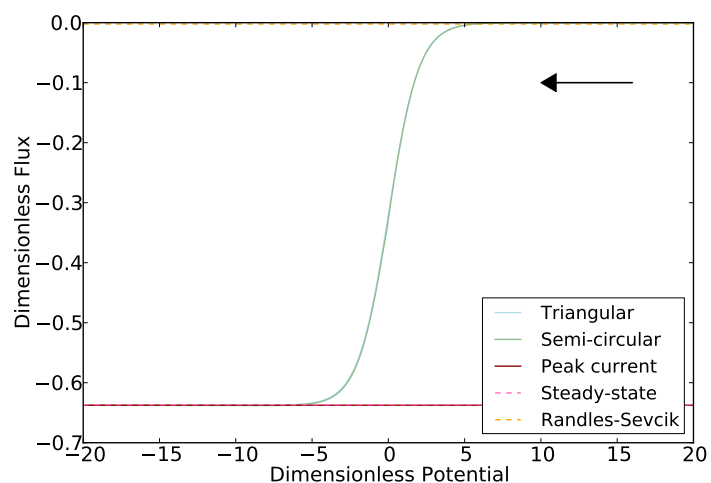

Figure 6: Voltammetric response using triangular and semi-circular potential waves at $\sigma=10^{-4}$. See caption of Figure 2 for further details.

From the analysis of Figures 2, 3, and 4, we show that under non-steady-state conditions the current response obtained from the semi-circular potential wave is significantly amplified especially at the potential where the singularity in the scan rate is observed, which in the above three cases is $\theta=0$, at the formal potential of the $A / B$ couple. We next therefore shift the potential window by changing the $\theta_{\text {shift }}$ value in order to examine the voltammetric behavior when the singularity in the scan rate occurs at a potential different from the formal potential. 


\subsection{Semi-circular potential sweeps: different $\theta_{\text {shift }}$ values}

In this section we investigate the effect of varying $\theta_{\text {shift }}$ values on the voltammetric response when using a semi-circular potential wave. As in section 3.1, we focus on scan rates $\sigma=10^{3}, \sigma=10^{1}$, and $\sigma=10^{-1}$. The formal potential of the $A / B$ couple is maintained at 0 .

Figure 7 shows the voltammetric response using the semi-circular potential wave at $\sigma=10^{3}$ with varying $\theta_{\text {shift }}$ values. $7 \mathrm{~A}$ corresponds to $\theta_{\text {shift }}=-4,7 \mathrm{~B}$ corresponds to $\theta_{\text {shift }}=-2,7 C$ corresponds to $\theta_{\text {shift }}=-1,7 D$ corresponds to $\theta_{\text {shift }}=0,7 E$ corresponds to $\theta_{\text {shift }}=2$, and $7 F$ corresponds to $\theta_{\text {shift }}=4$. In all figures, a sharp peak is visible and the potential at which it is observed equals the potential at which the singularity in the scan rate occurs, however, the amplification of the peak differs greatly depending on the $\theta_{\text {shift }}$ value. When the singularity occurs in the far negative region relative to the formal potential of the redox couple as in $7 A$, the peak current is low because much of the material is consumed at the electrode surface and thus increasing the scan rate has little effect on the electron transfer. As $\theta_{\text {shift }}$ is moved close to the formal potential such as in $7 B$ and $7 C$, we see the peak current increasing. In these regions, the system is much more sensitive to changes in the applied potential and thus show significant increase in the peak current. In Figure $7 E$, the singularity occurs at a higher potential relative to the formal potential, causing more materials to be consumed at that point resulting in the small sharp peak. However, as shown in Figure 1, the local scan rate rapidly decreases after the singularity which results in the decrease in the current after the sharp peak in 7 but then near the formal potential, a local absolute maximum is observed due to the diffusive current. In the far positive potential region relative to the formal potential as shown in Figure $7 F$, very little reaction is observed and thus the transiently infinitely fast scan rate does not influence the current response much and the peak current is controlled by diffusion. 

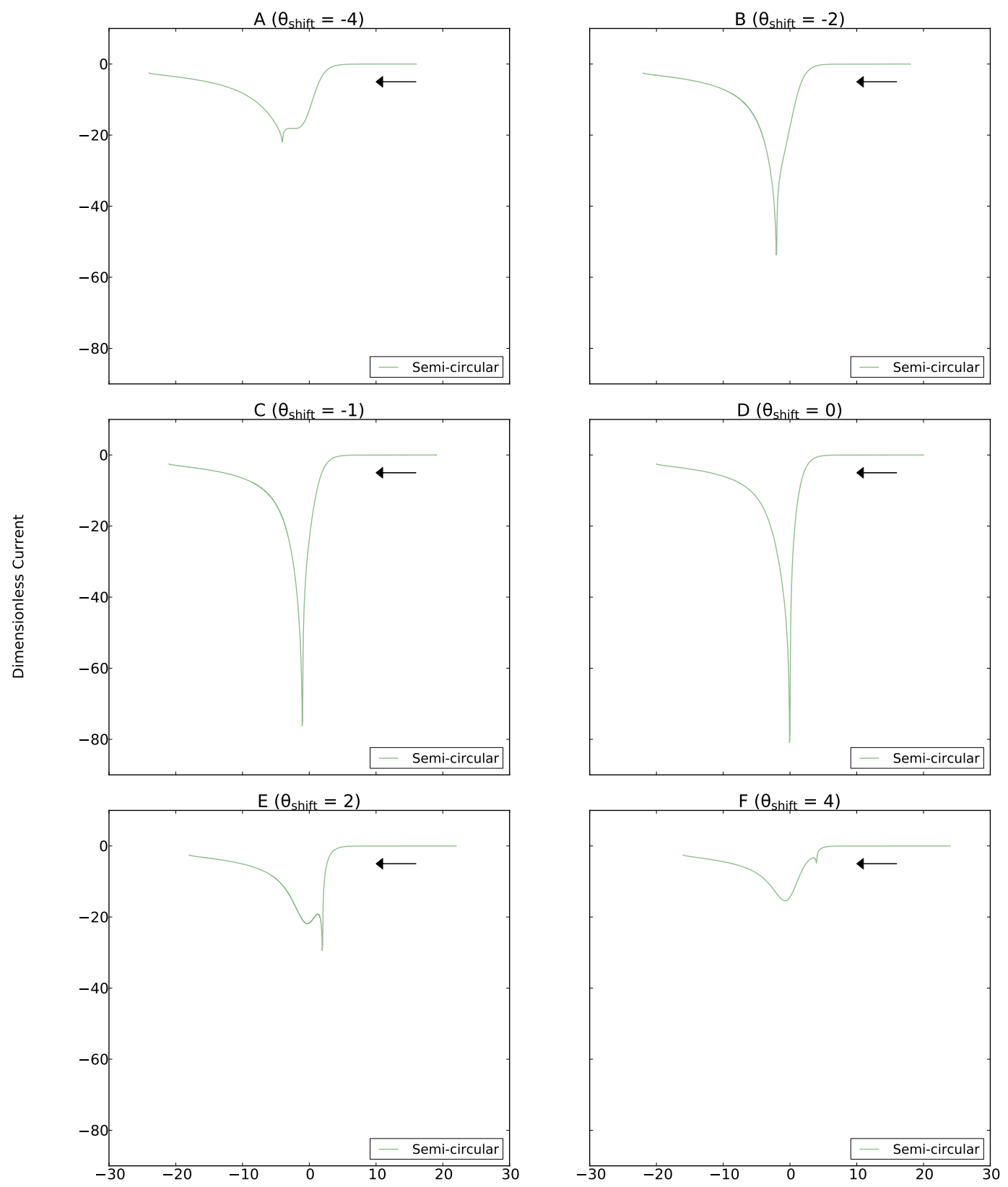

Dimensionless Potential

Figure 7: Voltammetric response using the semi-circular potential wave at $\sigma=10^{3}$ and $\theta_{f, A / B}^{0}=0$ with varying $\theta_{\text {shift }}$ values. $A$ corresponds to $\theta_{\text {shift }}=-4, B$ corresponds to $\theta_{\text {shift }}=-2, C$ corresponds to $\theta_{\text {shift }}=-1, D$ corresponds to $\theta_{\text {shift }}=0, E$ corresponds to $\theta_{\text {shift }}=2$, and $F$ corresponds to $\theta_{\text {shift }}=4$. The arrows indicate the direction of the sweep.

Figure 8 shows the voltammetric response using the semi-circular potential wave at $\sigma=10^{1}$. The results are reported using the same $\theta_{\text {shift }}$ values as in Figure 8 and very similar behavior is noted. When 
$\theta_{\text {shift }}$ is significantly negative relative to the formal potential, little effect of the singularity is visible whereas when $\theta_{\text {shift }}$ is close to the formal potential, significant increase in the peak current is seen. When the $\theta_{\text {shift }}$ is slightly positive relative to the formal potential two peaks occur, one resulting from the singularity of the potential wave and the other resulting from diffusion. In the far positive region where the electrode potential is not negative enough, the singularity has minimal effect on the current response. 

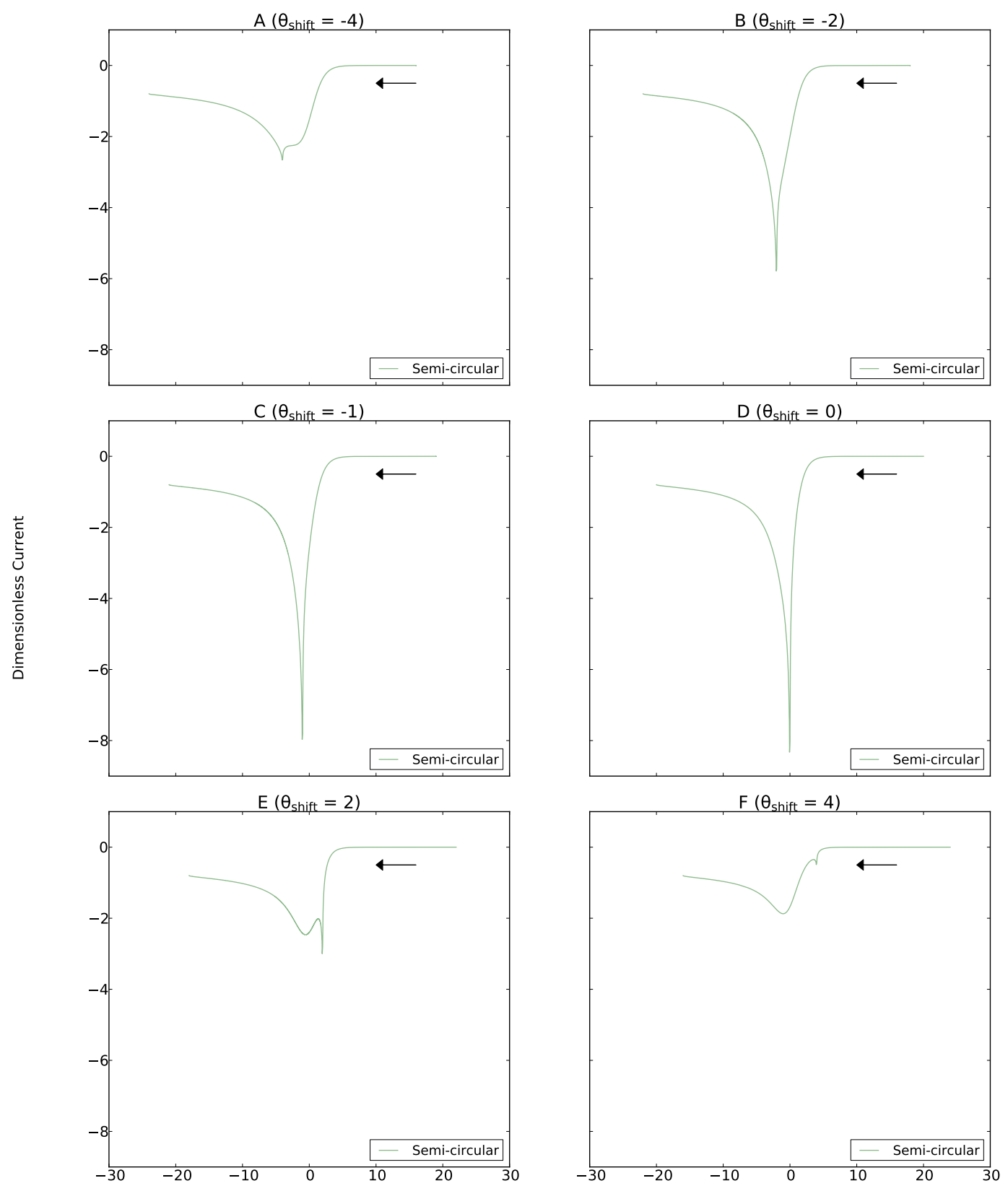

Dimensionless Potential

Figure 8: Voltammetric response using the semi-circular potential wave at $\sigma=10^{1}$ and $\theta_{f, A / B}^{0}=0$ with varying $\theta_{s h i f t}$ values. See caption of Figure 7 for further details.

Figure 9 shows the voltammetric response using the semi-circular potential wave at $\sigma=10^{-1}$. The results are again reported using the same $\theta_{\text {shift }}$ values as in Figures 7 and 8 . Since the system is 
near-steady-state due to the slow scan rate, the overall current is much lower but the unique current response arising from the semi-circular potential wave can still be identified. Figure 9 shows a very slight amplification of the peak current, which grows as $\theta_{\text {shift }}$ becomes closer to the formal potential as shown in $9 B$ and $9 C$. One interesting feature to note is that unlike in Figures 7 and 8 , the maximum peak current is observed at $\theta_{\text {shift }}=-1$. This is caused by the fact the instantaneously infinite scan rate of the potential wave coincides with the potential at which the diffusional peak current, i.e. the peak that arises from the diffusive mass transport along the concentration gradient rather than the temporarily accelerated scan rate, is observed, thereby enhancing the current response. This analysis becomes more apparent as we look at Figures $9 D, 9 E$, and $9 F .9 D$ is the same voltammogram as the semi-circular result in Figure 4 and shows a slight "dip". In Figures $9 B$ and $9 C$, a steep and rapid decrease in current is observed after the peak current until it levels off in the far negative potential region whereas in $9 D$, the current decreases significantly after $\theta_{\text {shift }}=0$ but then the curve becomes less steep before leveling off. This slight decrease in the steepness of the curve, which we call the "dip", is caused by the diffusional current. $9 F$ shows the diffusional current more prominently at around $\theta=-1$, and as $\theta_{\text {shift }}$ becomes increasingly positive, the sharp current response becomes less visible. 

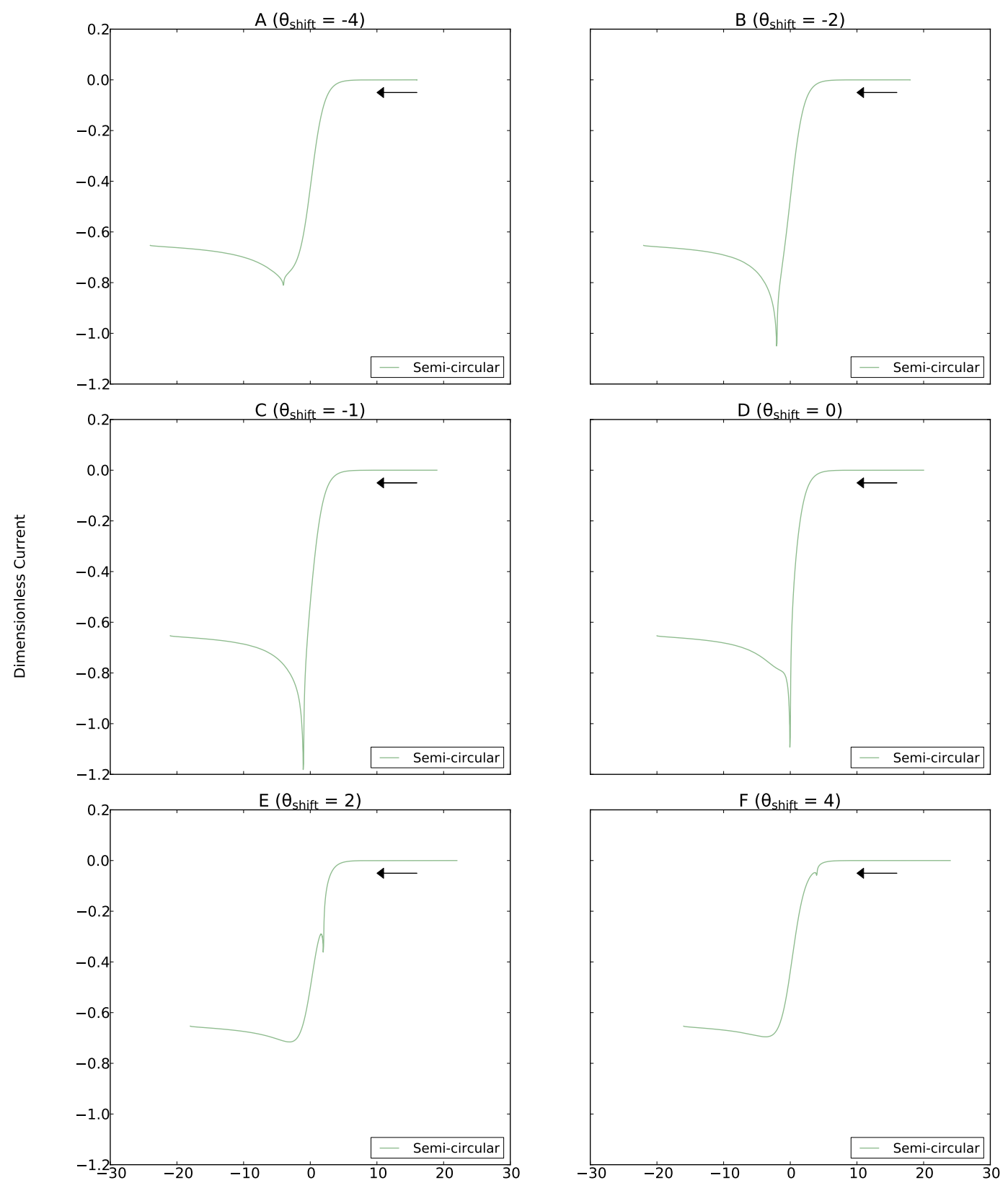

Dimensionless Potential

Figure 9: Voltammetric response using the semi-circular potential wave at $\sigma=10^{-1}$ and $\theta_{f, A / B}^{0}=0$ with varying $\theta_{\text {shift }}$ values. See caption of Figure 7 for further details.

The change in peak heights with respect to the $\theta_{\text {shift }}$ values is represented clearly in Figures 10a and 10b. Figure 10a shows the dimensionless peak heights plotted against the $\theta_{\text {shift }}$ values at which they 
are observed and Figure 10b shows the $\log _{10}$ of the dimensionless peak heights plotted against their respective $\theta_{\text {shift }}$ values. Both figures use the same color scheme where the light yellow dots represent results from $\sigma=10^{3}$, the light green dots represent results from $\sigma=10^{1}$, and the dark green dots represent results from $\sigma=10^{-1}$. As evident, peak height increases as $\theta_{\text {shift }}$ approaches 0 from reasons discussed earlier. However, from Figures 10a and 10b, it is unclear whether the absolute maximum occurs at $\theta_{\text {shift }}=0$ or somewhere between $\theta_{\text {shift }}=-1$ and $\theta_{\text {shift }}=0$. In order to determine the exact potential at which the absolute maximum peak height is observed, the $\theta_{\text {shift }}$ values must be more refined near the formal potential but for high scan rates such as $\sigma=10^{3}$, the absolute maximum peak height is assumed to be observed at a $\theta_{\text {shift }}$ value similar to that shown in our previous work ${ }^{4}$.

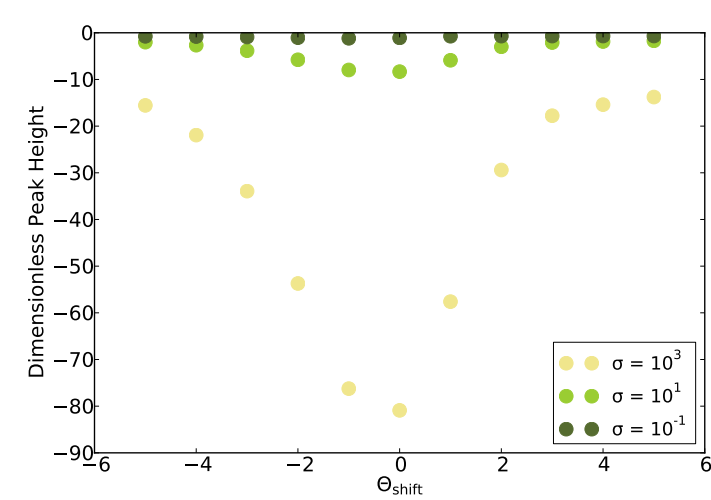

(a) Dimensionless peak heights against the $\theta_{\text {shift }}$ values at which they are observed for scan rates $10^{3}, 10^{1}$, and $10^{-1}$. The light yellow dots represent results from $\sigma=10^{3}$, the light green dots represent results from $\sigma=10^{1}$, and the dark green dots represent results from $\sigma=10^{-1}$.

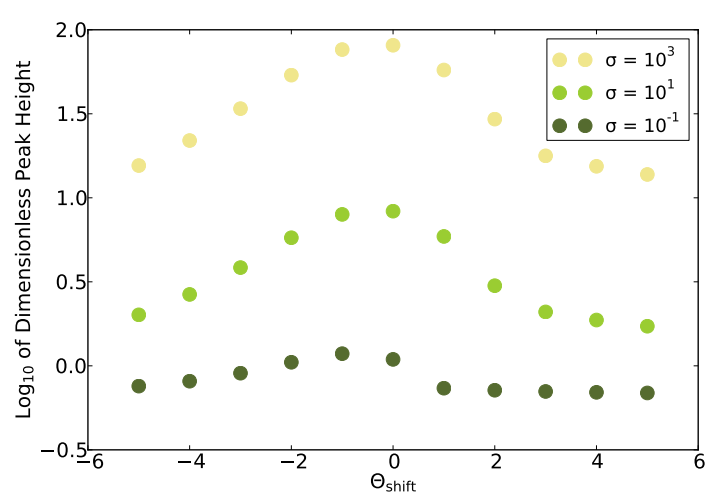

(b) $\log _{10}$ of the dimensionless peak heights against the $\theta_{\text {shift }}$ values at which they are observed for scan rates $10^{3}, 10^{1}$, and $10^{-1}$. The light yellow dots represent results from $\sigma=10^{3}$, the light green dots represent results from $\sigma=10^{1}$, and the dark green dots represent results from $\sigma=10^{-1}$.

Figure 10

From the results presented in Sections 3.1 and 3.2, the semi-circular potential wave exhibits similar advantages at a microelectrode as it does at a macroelectrode. The method introduced here gives the opportunity of amplifying a peak that would otherwise be too small to be seen in conventional triangular wave voltammetry, such as when the potentiostat's signal-to-noise ratio exceeds the peak heights in the conventional case and is thus ideal for electroanalysis. The technique may also be used to detect the presence of one pecies in a solution comprising multiple electroactive analytes where otherwise differential pulse or square wave voltammetry might be needed ${ }^{34 ; 35}$. In addition, the microelectrode response is important for the study of partially blocked electrodes ${ }^{36}$.

For our simulation, since $\theta_{f, A / B}^{0}=0$, the $\theta_{\text {shift }}$ expression is reduced to $\theta_{\text {shift }}=\theta_{\text {avg }}$. However in experimental practice, when analyzing a system containing unknown species, the only available parameter is the $\theta_{\text {avg }}$ value and thus the above analysis would be performed by repeatedly applying Equation (19) where $\theta_{\text {shift }}$ is replaced by $\theta_{\text {avg }}$ until an "infinite" peak or an absolute maximum peak is observed. Since, depending on $\sigma$, the infinite peak occurs at $\theta_{\text {shift }}=0$ or in its immediate vicinity, as shown through the above analysis, by determining the $\theta_{\text {avg }}$ value at which the absolute maximum current is observed, the formal potential of a species can be estimated. 


\section{Conclusions}

We have investigated a simple one-electron electrochemically reversible reaction using a semi-circular potential wave and shifted the potential window to study the resulting changes in voltammetric response at a microelectrode. The transiently infinitely fast scan rate at the midpoint of the potential wave results in an "infinite" peak current when the midpoint potential is at the formal potential of a redox system or in its immediate vicinity. The unique voltammetric features resulting from the semi-circular potential wave is suitable for electroanalysis and hence complements and in some situations can replace the conventional linear scan voltammetry.

Our analysis shows that the semi-circular potential wave exhibits similar advantages at microelectrodes as it does at macroelectrodes. This significantly broadens the applicability of this new voltammetric technique: Due to their advanced mass transport characteristics, their ease of handling, and their reusability, microelectrodes have de-facto become the standard electrode in electroanalysis. Since changing the voltammetric wave often only requires comparably small amendments to many electrochemical set-ups and microelectrodes are so commonly used, the presented work dramatically advances the availability of this new and exciting method to a wider scientific community.

\section{Acknowledgements}

The research leading to these results has received partial funding from the European Research Council under the European Union's Seventh Framework Programme (FP/2007-2013)/ERC Grant Agreement no. [320403].

\section{References}

[1] R. G. Compton, C. E. Banks, Understanding Voltammetry (2nd Edition), Imperial College Press, 2010 .

[2] Y. Uchida, E. Kätelhön, R. G. Compton, Cyclic voltammetry with non-triangular waveforms: Electrochemically reversible systems, Journal of Electroanalytical Chemistry 801 (2017) $381-387$.

[3] H. M. A. Amin, Y. Uchida, C. Batchelor-McAuley, E. Kätelhön, R. G. Compton, Non-triangular potential sweep cyclic voltammetry of reversible electron transfer: Experiment meets theory, Journal of Electroanalytical Chemistry 815 (2018) $24-29$.

[4] Y. Uchida, E. Kätelhön, R. G. Compton, Linear sweep voltammetry with non-triangular waveforms: New opportunities in electroanalysis, Journal of Electroanalytical Chemistry 818 (2018) 140 - 148.

[5] R. J. Forster, Microelectrodes: new dimensions in electrochemistry, Chem. Soc. Rev. 23 (1994) 289-297.

[6] M. Fleischmann, S. Pons, The behavior of microelectrodes, Analytical Chemistry 59 (24) (1987) 1391A-1399A.

[7] C. Amatore, C. Pebay, L. Thouin, A. Wang, J.-S. Warkocz, Difference between ultramicroelectrodes and microelectrodes: Influence of natural convection, Analytical Chemistry 82 (16) (2010) 69336939 . 
[8] C. Amatore, E. Maisonhaute, G. Simonneau, Ultrafast cyclic voltammetry: performing in the few megavolts per second range without ohmic drop, Electrochemistry Communications 2 (2) (2000) 81 -84 .

[9] R. G. Compton, J. C. Eklund, L. Nei, The application of microelectrodes to the study of photochemical reactions, Journal of Electroanalytical Chemistry 381 (1) (1995) 87 - 93.

[10] A. Lavacchi, U. Bardi, C. Borri, S. Caporali, A. Fossati, I. Perissi, Cyclic voltammetry simulation at microelectrode arrays with COMSOL multiphysics, Journal of Applied Electrochemistry 39 (11) (2009) 2159.

[11] H. T. H. Chan, E. Kätelhön, R. G. Compton, Voltammetry using multiple cycles: Porous electrodes, Journal of Electroanalytical Chemistry 799 (2017) 126 - 133.

[12] M. C. Henstridge, E. Laborda, E. J. Dickinson, R. G. Compton, Redox systems obeying MarcusHush-Chidsey electrode kinetics do not obey the Randles-Ševčík equation for linear sweep voltammetry, Journal of Electroanalytical Chemistry 664 (2012) $73-79$.

[13] E. J. F. Dickinson, J. G. Limon-Petersen, N. V. Rees, R. G. Compton, How much supporting electrolyte is required to make a cyclic voltammetry experiment quantitatively "diffusional"? A theoretical and experimental investigation, The Journal of Physical Chemistry C 113 (25) (2009) $11157-11171$.

[14] C. Amatore, A. Oleinick, I. Svir, Theoretical analysis of microscopic ohmic drop effects on steadystate and transient voltammetry at the disk microelectrode: A quasi-conformal mapping modeling and simulation, Analytical Chemistry 80 (21) (2008) 7947-7956.

[15] S. Ching, R. Dudek, E. Tabet, Cyclic voltammetry with ultramicroelectrodes, Journal of Chemical Education 71 (7) (1994) 602.

[16] J. K. Novev, R. G. Compton, Natural convection effects in electrochemical systems, Current Opinion in Electrochemistry 7 (2018) $118-129$.

[17] C. N. Reilley, G. W. Everett, R. Johns, Voltammetry at constant current: Experimental evaluation, Analytical Chemistry 27 (4) (1955) 483-491.

[18] K. Ngamchuea, S. Eloul, K. Tschulik, R. G. Compton, Advancing from rules of thumb: Quantifying the effects of small density changes in mass transport to electrodes. understanding natural convection, Analytical Chemistry 87 (14) (2015) 7226-7234.

[19] X. Gao, J. Lee, H. S. White, Natural convection at microelectrodes, Analytical Chemistry 67 (9) (1995) 1541-1545.

[20] R. G. Compton, E. Laborda, K. R. Ward, Understanding Voltammetry: Simulation of Electrode Processes, Imperial College Press, 2013.

[21] J. Crank, The Mathematics of Diffusion, Oxford University Press, 1979.

[22] K. R. Ward, N. S. Lawrence, R. S. Hartshorne, R. G. Compton, Modelling the steady state voltammetry of a single spherical nanoparticle on a surface, Journal of Electroanalytical Chemistry 683 (2012) $37-42$. 
[23] E. J. F. Dickinson, I. Streeter, R. G. Compton, Theory of diffusion to an annular microband electrode, Journal of Electroanalytical Chemistry 625 (1) (2009) $40-46$.

[24] R. Guidelli, R. G. Compton, J. M. Feliu, E. Gileadi, J. Lipkowski, W. Schmickler, S. Trasatti, Definition of the transfer coefficient in electrochemistry (IUPAC recommendations 2014) 86 (2014) 259-262.

[25] R. Guidelli, R. G. Compton, J. M. Feliu, E. Gileadi, J. Lipkowski, W. Schmickler, S. Trasatti, Defining the transfer coefficient in electrochemistry: An assessment (IUPAC technical report) 86 (2014) 245-258.

[26] A. J. Bard, L. R. Faulkner, Electrochemical Methods: Fundamentals and Applications, John Wiley \& Sons Inc, 2000.

[27] J. Heinze, Diffusion processes at finite (micro) disk electrodes solved by digital simulation, Journal of Electroanalytical Chemistry and Interfacial Electrochemistry 124 (1) (1981) 73 - 86.

[28] S. Eloul, R. G. Compton, Implementing high performance voltammetry simulation using the implicit parallel algorithm, Journal of Electroanalytical Chemistry 771 (2016) 50 - 55.

[29] E. Kätelhön, R. G. Compton, Testing and validating electroanalytical simulations, Analyst 140 (2015) 2592-2598.

[30] J. E. B. Randles, A cathode ray polarograph. Part II.-The current-voltage curves, Trans. Faraday Soc. 44 (1948) 327-338.

[31] A. Ševčík, Oscillographic polarography with periodical triangular voltage, Collect. Czech. Chem. Commun. 13 (1948) 349-377.

[32] F. G. Chevallier, O. V. Klymenko, L. Jiang, T. G. J. Jones, R. G. Compton, Mathematical modelling and numerical simulation of adsorption processes at microdisk electrodes, Journal of Electroanalytical Chemistry 574 (2) (2005) 217 - 237.

[33] A. M. Bond, K. B. Oldham, C. G. Zoski, Steady-state voltammetry, Analytica Chimica Acta 216 (1989) $177-230$

[34] R. T. Kachoosangi, R. G. Compton, A simple electroanalytical methodology for the simultaneous determination of dopamine, serotonin and ascorbic acid using an unmodified edge plane pyrolytic graphite electrode, Analytical and Bioanalytical Chemistry 387 (8) (2007) 2793-2800.

[35] A. Molina, J. González, Pulse Voltammetry in Physical Electrochemistry and Electroanalysis: Theory and Applications (Monographs in Electrochemistry), Springer, 2016.

[36] B. A. Brookes, T. J. Davies, A. C. Fisher, R. G. Evans, S. J. Wilkins, K. Yunus, J. D. Wadhawan, R. G. Compton, Computational and experimental study of the cyclic voltammetry response of partially blocked electrodes. Part 1. nonoverlapping, uniformly distributed blocking systems, Journal of Physical Chemistry B 107 (7) (2003) 1616-1627. 